\title{
CHARACTERIZATION OF OIL AND GAS RESERVOIR HETEROGENEITY
}

\author{
Cooperative Agreement DE-FG07-901D12839
}

DOE/ID/12839--9

Petroleum Development Laboratory

DE93 003304 University of Alaska Fairbanks

437 Duckering Building

Fairbanks, AK 99775-1260

\section{DISCLAIMER}

This report was prepared as an account of work sponsored by an agency of the United States Government. Neither the United States Gnvernment nor any agency thereof, nor any of their employees, makes any warranty, express or implied, or assumes any legal liability or responsibility for the accuracy, completeness, or usefulness of any information, apparatus, product, or process disclosed, or represents that its use would not infringe privately owned rights. Reference herein to any specific commercial product, process, or service by trade name, trademark, manufacturer, or otherwise does not necessarily constitute or imply its endorsement, recommendation, or favoring by the United States Government or any agency thereof. The views and opinions of authors expressed herein do not necessarily state or reflect those of the United States Government or any agency thereof.

rrincipal investigator:

G. D. Sharma

Project Manager:

Idaho Operations Office

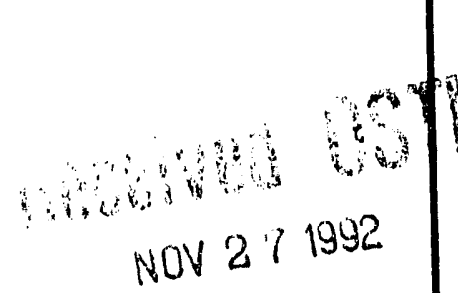

Reporting Period: July 1, 1992 - September 30, 1992

\section{OBJECTIVE}

The ultimate objective of this cooperative research project is to characterize Alaskan petroleum reservoirs in terms of their reserves, physical and chemical properties, geologic configuration in relation to lithofacies and structure, and development potential.

The project has two tasks: Task 1 is a geological description of the reservoirs including petruphysical properties, i.e., porosity, permeability, permeability variation, formation depth, temperature, and net pay, facies changes and reservoir structures as drawn from cores, well logs, and other geological data. Task 2 is reservoir fluid characterization-determination of physical properties of reservoir fluids including density, viscosity, phase distributions and composition as well as petrogenesissource rock identification; and the study of asphaltene precipitation for Alaskan crude oils. 


\section{SUMMARY OF TECHNICAL PROGRESS}

\section{WELL LOG ANALYSIS OF KUPARUK FIELD, NORTH SLOPE, ALASKA}

During the last quarter, preliminary analysis of approximately 50 Kuparuk Field wells was undertaken at PDL. Figure 1 shows locations of wells which have been analyzed or are currently being analyzed. The analysis is focusing on the Lower Cretaceous Kuparuk River Upper anu' Lower Sands, which are the main producing Kuparuk intervals (Figure 2). Calculation of porosity, water saturation, gross and net pay, and shale volume at each well has been completed and results are being stored for future mapping and reservoir description. Tables 1 and 2 list petrophysical values computed to date in Kuparuk Field wells. The analysis was initiated in the southern portion of the field and is currently being carried north.

Plots of the Kuparuk Upper and Lower Sands similar to those in Figures 3 and 4 have been generated as a means of determining field-wide patterns in log character (and corresponding rock character). Figures 5 and 6 , depicting results of petrophysicai analysis of the Upper and Lower Sands, indicate the presence of hydrocarbons. Cross sections like those in Figures 7 and 8 are also being generated in the effort to better delineate areal continuity of the producing sands and to isolate faults and structural trends.

Literature discussing Kuparuk River Field indicates the structure and stratigraphy of the sands to be coinplex, as indicated in Figure 9. Extensive faulting as show in Figure 10 has played an important role in development of hydrocarbon migration patterns. An example of stratigraphic complexity within the Lower Sand interval is 
exhibited where progressively younger " $A$ " sands pinchout southward across the field (Figure 11).

As input of well log data into the Kuparuk Field database continues during the next quarter, analysis of the Upper and Lower Sand intervals will be intensified in order to isolate and identify individual sand members within each interval. Placement of faults and identification of structural elements will be incorporated in the reservoir

description. Stratigraphy of the various sands will be analyzed, with efforts being concentrated on the interrelationship of faulting and sand thickening, thinning, and pinchouts. Log character will be evaluated to determine environments of deposition and reservoir sand quality. In addition, contour maps and three-dimensional plots will be generated as part of the reservoir description.

\section{REFERENCE}

Masterson, W.D., and C.E. Paris: "Depositional History and Reservoir Description of the Kuparuk River Formation, North Slope, Alaska," Alaskan North Slope Geology, Vol. 1, edited by Irv Tailbur and Paul Weirner, published by the Pacific Section, Society of Economic Paleontologists and Mineralogists, Bakersfield, CA, and the Alaskan Geology Society, Anchorage, AK (1987). 


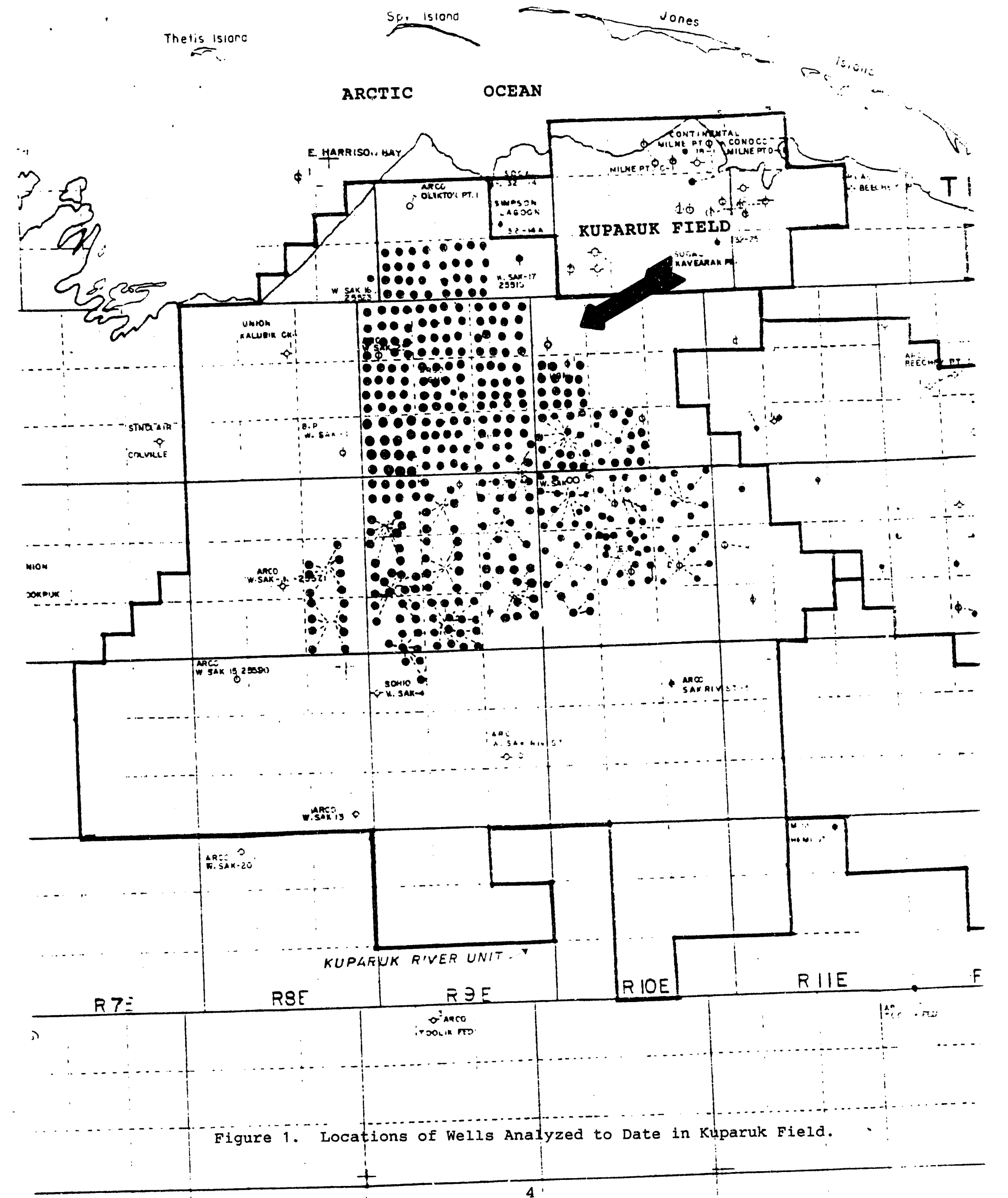




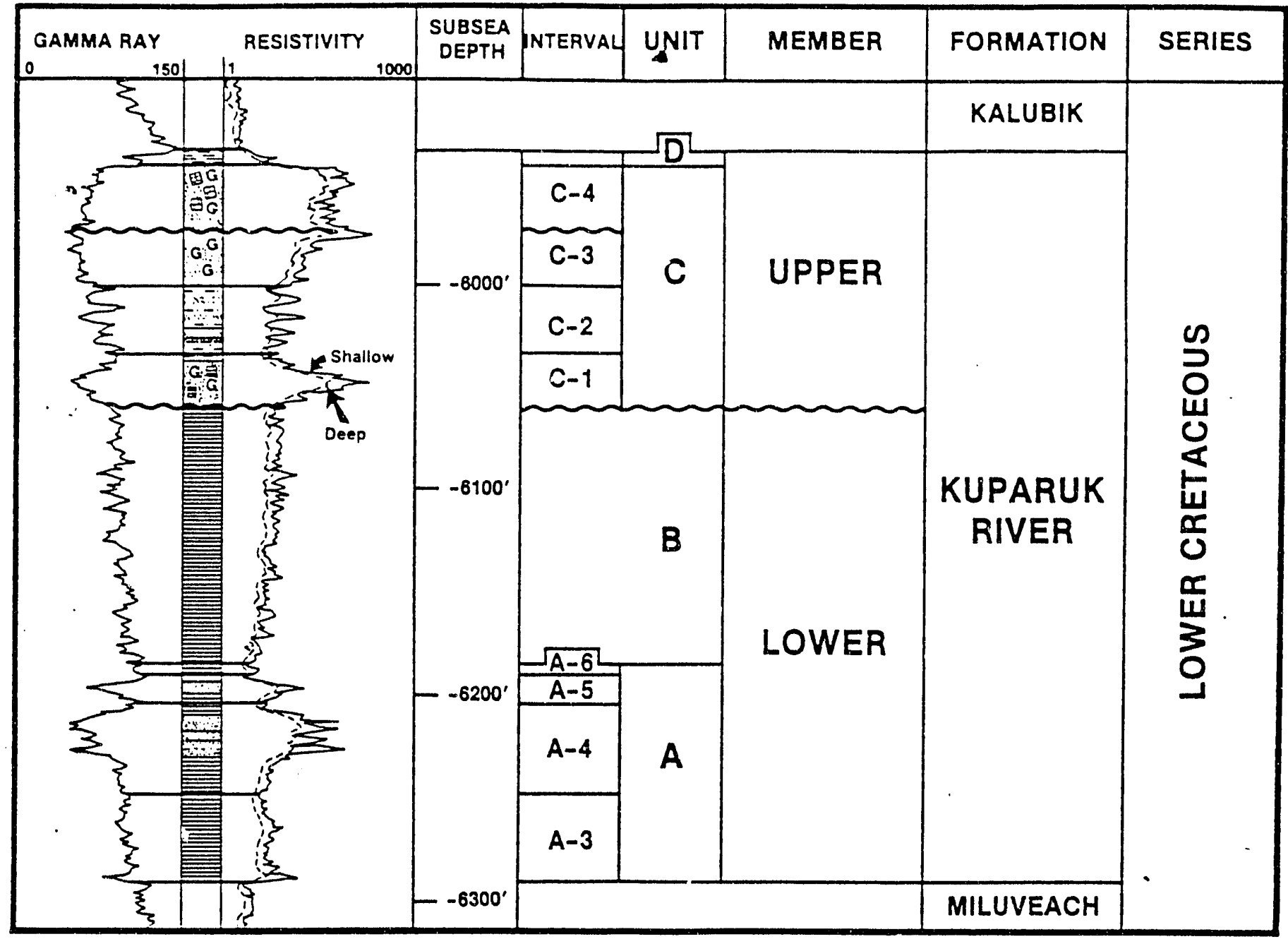

LEGEND

\begin{tabular}{lll}
$\square$ SANDSTONE & SILTSTONE & SIDERITE CEMENT \\
$\square$ MUDSTONE & $G_{G}$ GLAUCONITE & SILRBEDDED SANDSTONE, \\
\hline
\end{tabular}

Figure 2. Stratigraphy of Kuparuk Formation

Showing Upper and Lower Sand Members (Masterson and Paris). 
Table 1. Upper Sand Petrophysical Values at Kuparuk Field

\begin{tabular}{|c|c|c|c|c|c|}
\hline $\begin{array}{c}\text { UPPER } \\
\text { SAND }\end{array}$ & $\begin{array}{c}\text { GROSS } \\
\text { THICKNESS } \\
(\mathrm{ft})\end{array}$ & $\begin{array}{c}\text { NET PAY } \\
(\mathrm{ft})\end{array}$ & $\phi(\%)$ & SW (\%) & $\begin{array}{c}\text { VOLUME } \\
\text { OF SHALE } \\
(\%)\end{array}$ \\
\hline $1 \mathrm{~A}-11$ & 40 & 15 & 25 & 35 & \\
\hline $1 \mathrm{~A}-14$ & 120 & $(16)$ & $(25)$ & $(34)$ & \\
\hline $1 \mathrm{~B} 7$ & 43 & 1 & 19 & 78 & \\
\hline $1 \mathrm{C} 2$ & 122 & 117 & 22 & 34 & 15 \\
\hline $1 \mathrm{C5}$ & 157 & 137 & 26 & 35 & 18 \\
\hline $1 \mathrm{D} 2$ & 80 & 17 & 25 & 54 & \\
\hline $1 \mathrm{D} 4$ & 122 & 109 & 34 & 18 & 58 \\
\hline $1 \mathrm{D} 6$ & 48 & 15 & 17 & $2 ?$ & 26 \\
\hline $1 \mathrm{G} 7$ & 51 & 41 & 19 & 53 & 20 \\
\hline $1 \mathrm{G} 13$ & 94 & 47 & 16 & 28 & \\
\hline $1 \mathrm{H3}$ & $(108)$ & $(19)$ & $(23)$ & $(42)$ & \\
\hline $1 \mathrm{H} 6$ & 38 & 0 & 27 & 90 & \\
\hline $1 \mathrm{H} 7$ & 42 & 9 & 26 & 85 & \\
\hline $1 \mathrm{H} 13$ & 118 & 22 & 20 & 32 & \\
\hline $1 \mathrm{~L} 8$ & 51 & 38 & 23 & 35 & \\
\hline $1 \mathrm{Q} 11$ & 65 & 41 & 18 & 30 & \\
\hline $1 \mathrm{Q} 15$ & 58 & 44 & 13 & 70 & 23 \\
\hline $1 \mathrm{Y} 16$ & 43 & 35 & 17 & 46 & \\
\hline $2 \mathrm{~A} 5$ & 36 & 36 & 21 & 27 & 11 \\
\hline $2 \mathrm{~B} 3$ & 133 & 57 & 17 & 54 & 26 \\
\hline $2 \mathrm{D} 11$ & 26 & 6 & 15 & 53 & 56 \\
\hline $2 \mathrm{D} 12$ & 20 & 15 & 16 & 42 & 14 \\
\hline $2 \mathrm{E} 11$ & 27 & 26 & 18 & 65 & 18 \\
\hline $2 \mathrm{~F} 1$ & 120 & 120 & 29 & 25 & 3 \\
\hline $2 \mathrm{~F} 2$ & 12 & 12 & 17 & 42 & 27 \\
\hline $2 \mathrm{~F} 15$ & 119 & 60 & 23 & 24 & 9 \\
\hline
\end{tabular}




\begin{tabular}{|c|c|c|c|c|c|}
\hline $\begin{array}{c}\text { UPPER } \\
\text { SAND }\end{array}$ & $\begin{array}{c}\text { GROSS } \\
\text { THICKNESS } \\
(\mathrm{ft})\end{array}$ & $\begin{array}{c}\text { NET PAY } \\
(\mathrm{ft})\end{array}$ & $\Phi(\%)$ & Sw (\%) & $\begin{array}{c}\text { VOLUME } \\
\text { OF SHALE } \\
(\%)\end{array}$ \\
\hline $2 \mathrm{G} 6$ & 120 & 2 & 13 & 35 & 28 \\
\hline $2 \mathrm{~h} 13$ & 154 & 115 & 19 & 39 & 20 \\
\hline $2 \mathrm{~h} 15$ & 120 & 4 & 12 & 44 & 27 \\
\hline $2 \mathrm{u} 1$ & 74 & 62 & 14 & 33 & 4 \\
\hline $2 \mathrm{u} 6$ & 45 & 37 & 16 & 40 & 1 \\
\hline $2 \mathrm{u} 16$ & 67 & 63 & 23 & 15 & 14 \\
\hline $2 \mathrm{v} 14$ & 51 & 49 & 18 & 23 & 4 \\
\hline $2 \mathrm{w} 2$ & 58 & 49 & 17 & 29 & 11 \\
\hline $2 \mathrm{w} 7$ & 60 & 59 & 21 & 20 & 12 \\
\hline $2 \mathrm{z} 7$ & 51 & 47 & 19 & 40 & 17 \\
\hline $2 \times 2$ & 129 & 108 & 21 & 41 & 19 \\
\hline $2 \mathrm{z} 15$ & 87 & 71 & 26 & 15 & 19 \\
\hline $3 \mathrm{a} 9$ & 90 & 79 & 18 & 42 & 17 \\
\hline $3 \mathrm{~b} 5$ & 40 & 8 & 14 & 44 & 37 \\
\hline $3 \mathrm{~b} 14$ & 150 & 150 & 21 & 41 & 11 \\
\hline $3 \mathrm{n} 12$ & 64 & 15 & 15 & 39 & 32 \\
\hline $3 \mathrm{n} 13$ & 35 & 29 & 15 & 54 & 19 \\
\hline
\end{tabular}


Table 2. Lower Sand Petrophysical Values at Kuparuk Field

\begin{tabular}{|c|c|c|c|c|c|}
\hline $\begin{array}{l}\text { LOWER } \\
\text { SAND }\end{array}$ & $\begin{array}{c}\text { GROSS } \\
\text { THICKNESS } \\
\text { (ft) }\end{array}$ & $\begin{array}{l}\text { NET PAY } \\
\text { (ft) }\end{array}$ & $\phi(\%)$ & Sw $(\%)$ & $\begin{array}{l}\text { VOLUME } \\
\text { OF SHALE } \\
(\%)\end{array}$ \\
\hline $1 \mathrm{~A}-11$ & 46 & 22 & 28 & 31 & \\
\hline $1 \mathrm{~A}-14$ & 92 & $(16)$ & (17) & 36 & \\
\hline $1 \mathrm{~B} 7$ & 47 & 4 & 29 & 38 & \\
\hline $1 C 2$ & 16 & 12 & 25 & 46 & 10 \\
\hline $1 C 5$ & 107 & 77. & 25 & 49 & 49 \\
\hline 1D2 & 42 & 0 & 21 & 77 & \\
\hline 1D4 & 33 & 33 & 34 & 18 & 58 \\
\hline 106 & 33 & & & & \\
\hline \multicolumn{6}{|l|}{$1 \mathrm{G7}$} \\
\hline $1 \mathrm{G} 13$ & 118 & 22 & 20 & 33 & \\
\hline $1 \mathrm{H3}$ & (105) & (21) & $(22)$ & (50) & \\
\hline $1 \mathrm{H} 6$ & 119 & 0 & 23 & 93 & \\
\hline $1 \mathrm{H7}$ & 50 & 25 & 28 & 35 & \\
\hline $1 \mathrm{H} 13$ & 118 & 22 & 20 & 32 & \\
\hline $1 L 8$ & 94 & 1 & 25 & 66 & \\
\hline 1Q11 & $(120)$ & (37) & (22) & (26) & \\
\hline $1 \mathrm{Q} 15$ & 90 & 80 & 21 & 32 & 18 \\
\hline \multicolumn{6}{|l|}{$1 Y 16$} \\
\hline $2 \mathrm{~A} 5$ & 121 & 721 & 19 & 35 & 17 \\
\hline $2 \mathrm{B3}$ & (45) & (30) & (20) & (43) & (15) \\
\hline 2D11 & 50 & 50 & 17 & 45 & 23 \\
\hline $2 \mathrm{D} 12$ & 83 & 83 & 22 & 32 & 12 \\
\hline $2 \mathrm{E} 11$ & 103 & 103 & 22 & 54 & 22 \\
\hline \multicolumn{6}{|l|}{$2 \mathrm{~F} 1$} \\
\hline $2 F 2$ & 120 & 120 & 20 & 32 & 26 \\
\hline $2 \mathrm{~F} 15$ & 14 & 1 & 20 & 39 & 11 \\
\hline
\end{tabular}




\begin{tabular}{|c|c|c|c|c|c|}
\hline $\begin{array}{c}\text { LOWER } \\
\text { SAND }\end{array}$ & $\begin{array}{c}\text { GROSS } \\
\text { THICKNESS } \\
(\mathrm{ft})\end{array}$ & $\begin{array}{c}\text { NET PAY } \\
(\mathrm{ft})\end{array}$ & $\phi(\%)$ & $S W(\%)$ & $\begin{array}{c}\text { VOLUME } \\
\text { OF SHALE } \\
(\%)\end{array}$ \\
\hline $2 \mathrm{G} 6$ & 127 & 115 & 15 & 10 & 22 \\
\hline $2 \mathrm{~h} 13$ & 104 & 100 & 20 & 37 & 21 \\
\hline $2 \mathrm{~h} 15$ & 127 & 115 & 15 & 10 & 22 \\
\hline $2 \mathrm{u} 1$ & 121 & 110 & 17 & 38 & 38 \\
\hline $2 \mathrm{u} 6$ & 124 & 123 & 21 & 49 & 15 \\
\hline $2 \mathrm{u} 16$ & 39 & 39 & 20 & 22 & 17 \\
\hline $2 \mathrm{v} 14$ & 134 & 133 & 20 & 39 & 16 \\
\hline $2 \mathrm{w} 2$ & 113 & 102 & 18 & 32 & 19 \\
\hline $2 \mathrm{w} 7$ & 115 & 91 & 17 & 32 & 26 \\
\hline $2 \mathrm{z} 7$ & 62 & 40 & 18 & 20 & 12 \\
\hline $2 \times 2$ & 92 & 92 & 20 & 23 & 8 \\
\hline $2 \mathrm{z} 15$ & 119 & 37 & 19 & 29 & 29 \\
\hline $3 \mathrm{a} 9$ & 109 & 108 & 22 & 32 & 14 \\
\hline $3 \mathrm{~b} 5$ & 96 & 82 & 22 & 38 & 11 \\
\hline $3 \mathrm{~b} 14$ & 137 & 75 & 25 & 46 & 6 \\
\hline $3 \mathrm{n} 12$ & 120 & 120 & 17 & 39 & 31 \\
\hline $3 \mathrm{n} 13$ & 109 & 109 & 19 & 29 & 30 \\
\hline
\end{tabular}


Figure 3. Upper and Lower Sands in Well 2w2.

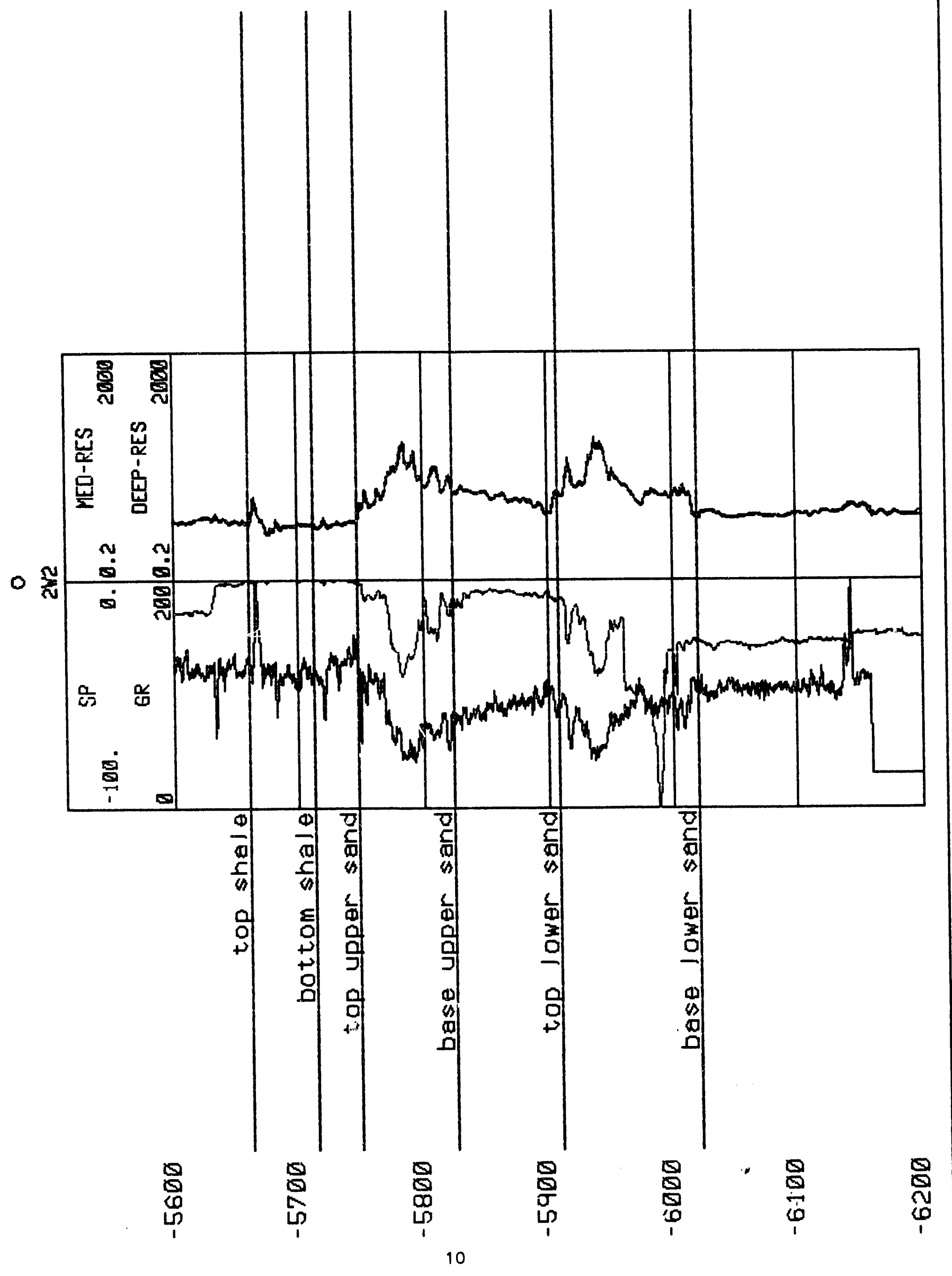


Figure 4. Upper and Lower Sands in Well 1Q-15.

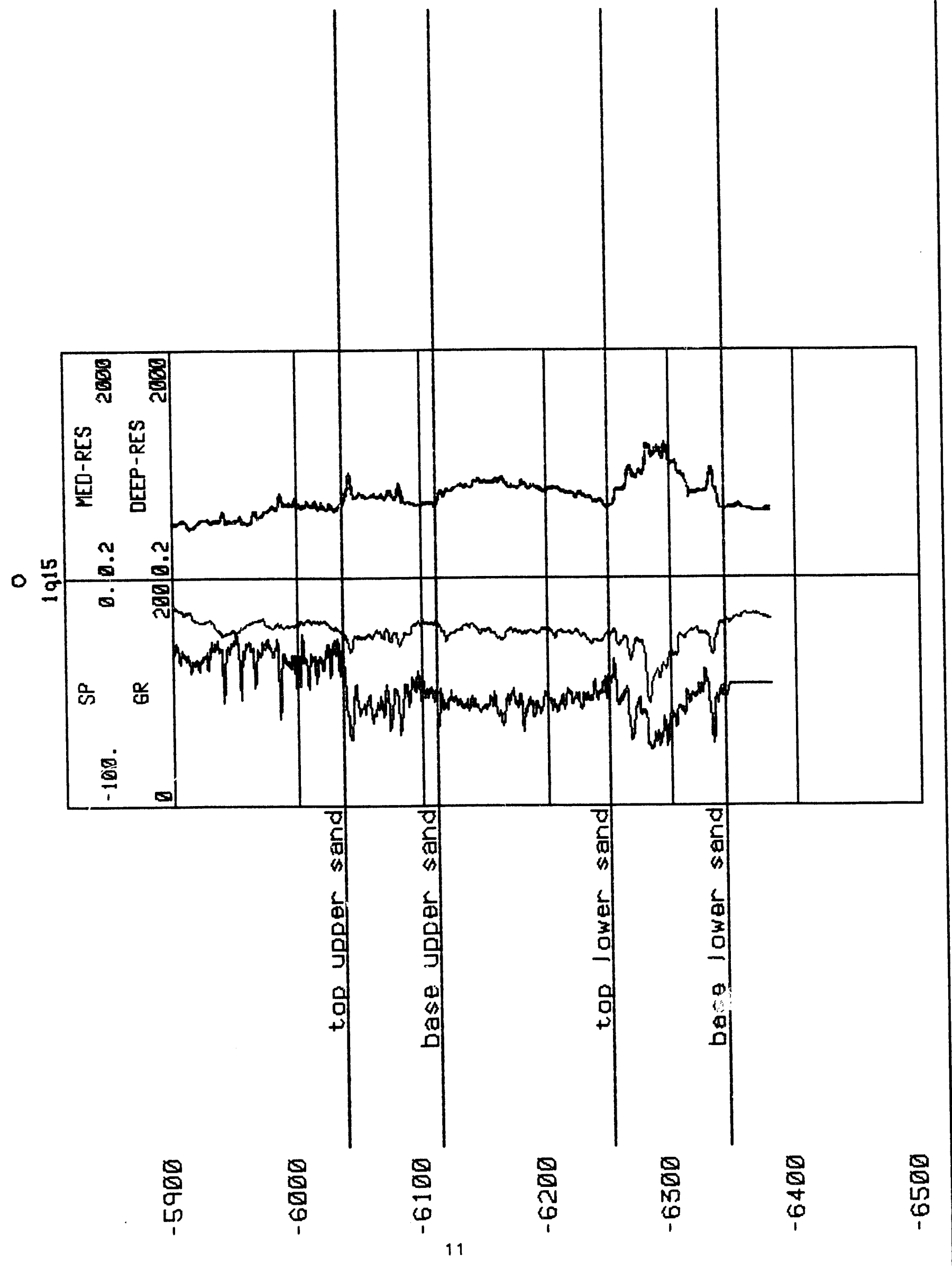


Figure 5. Analysis Plot of Well 2w2.

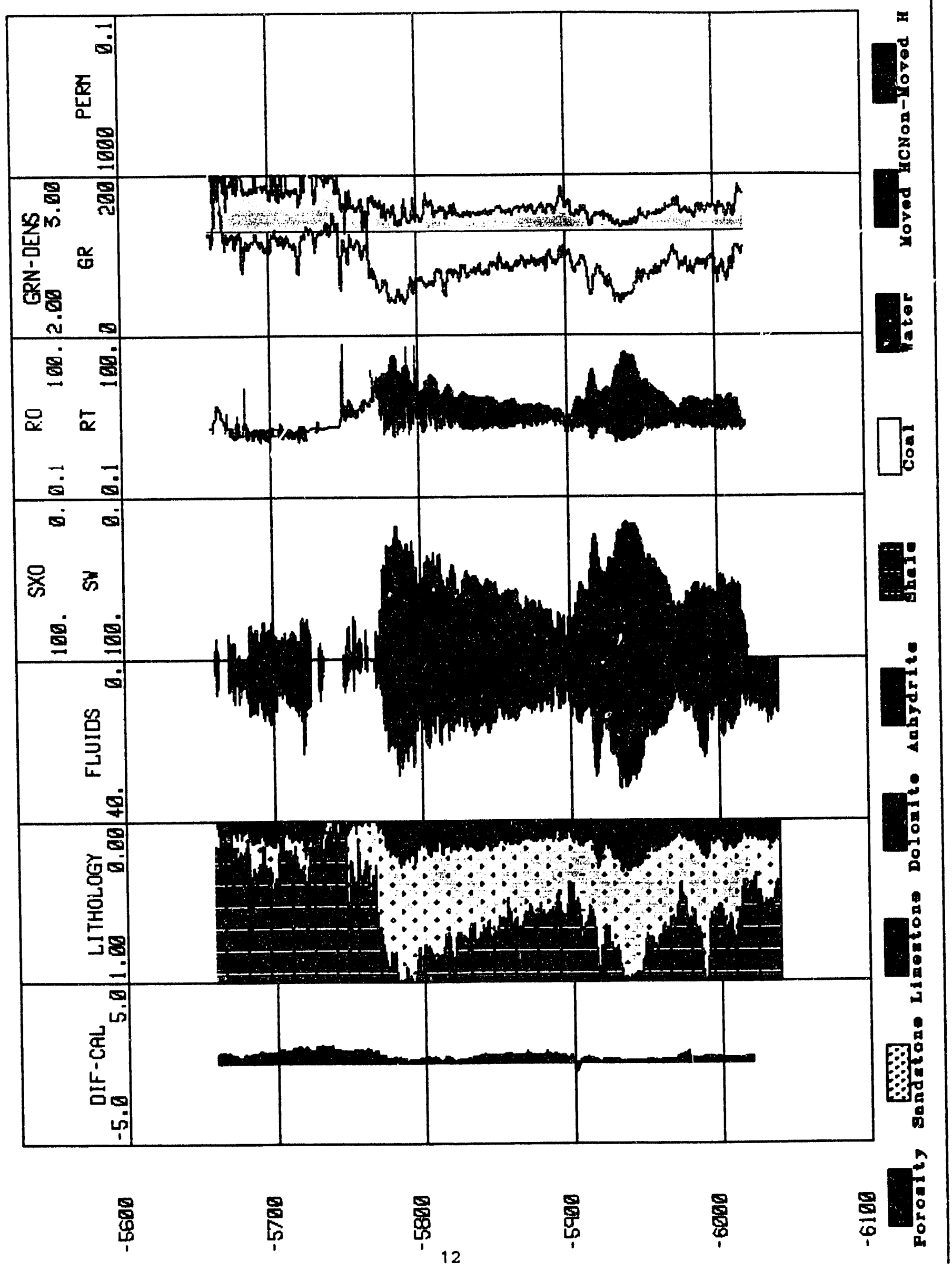


Figure 6. Analysis Plot of Well 1Q-15.

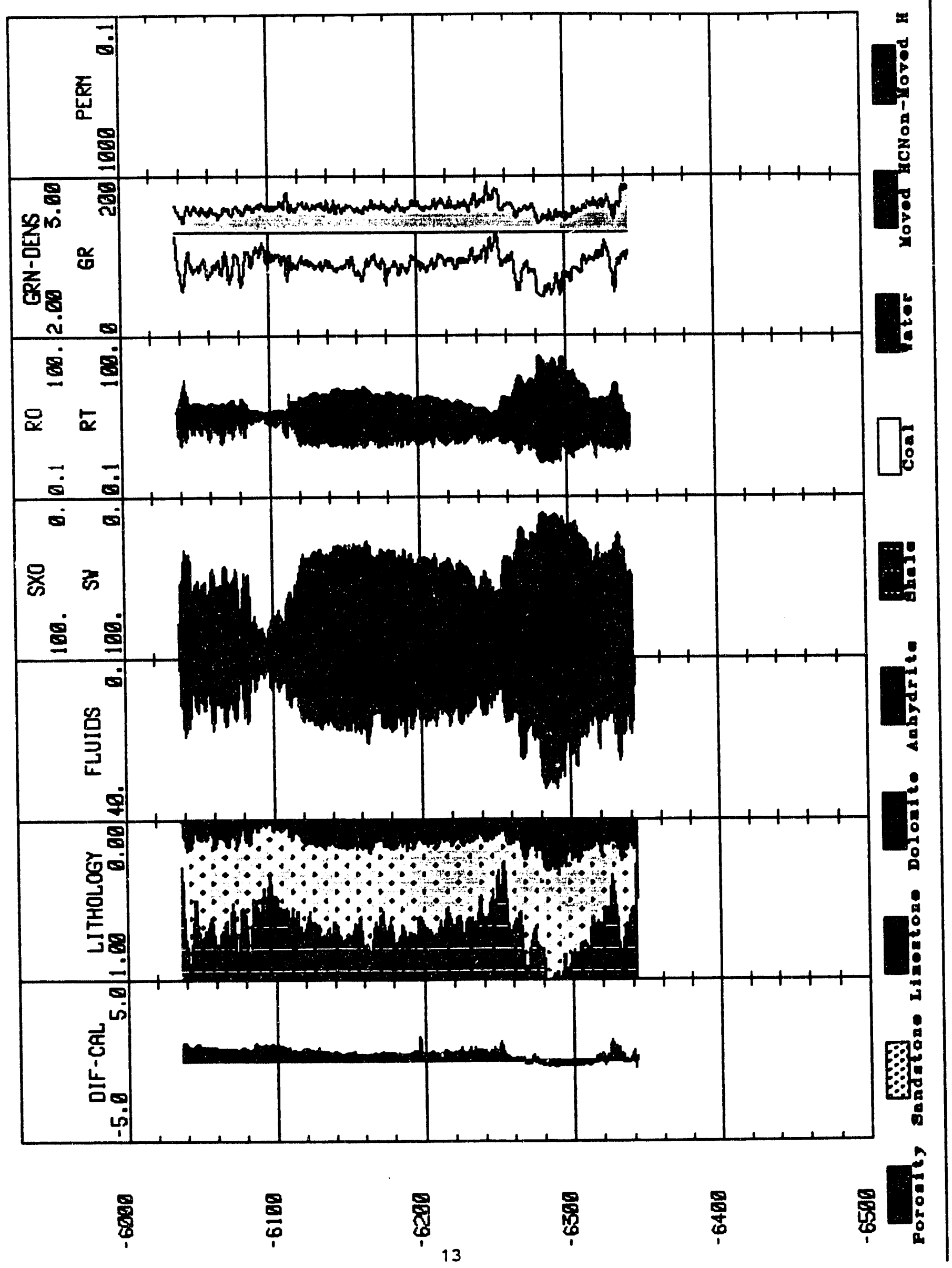


Figure 7. Cross Section Showing Northerly Dip Across Kuparuk Field.

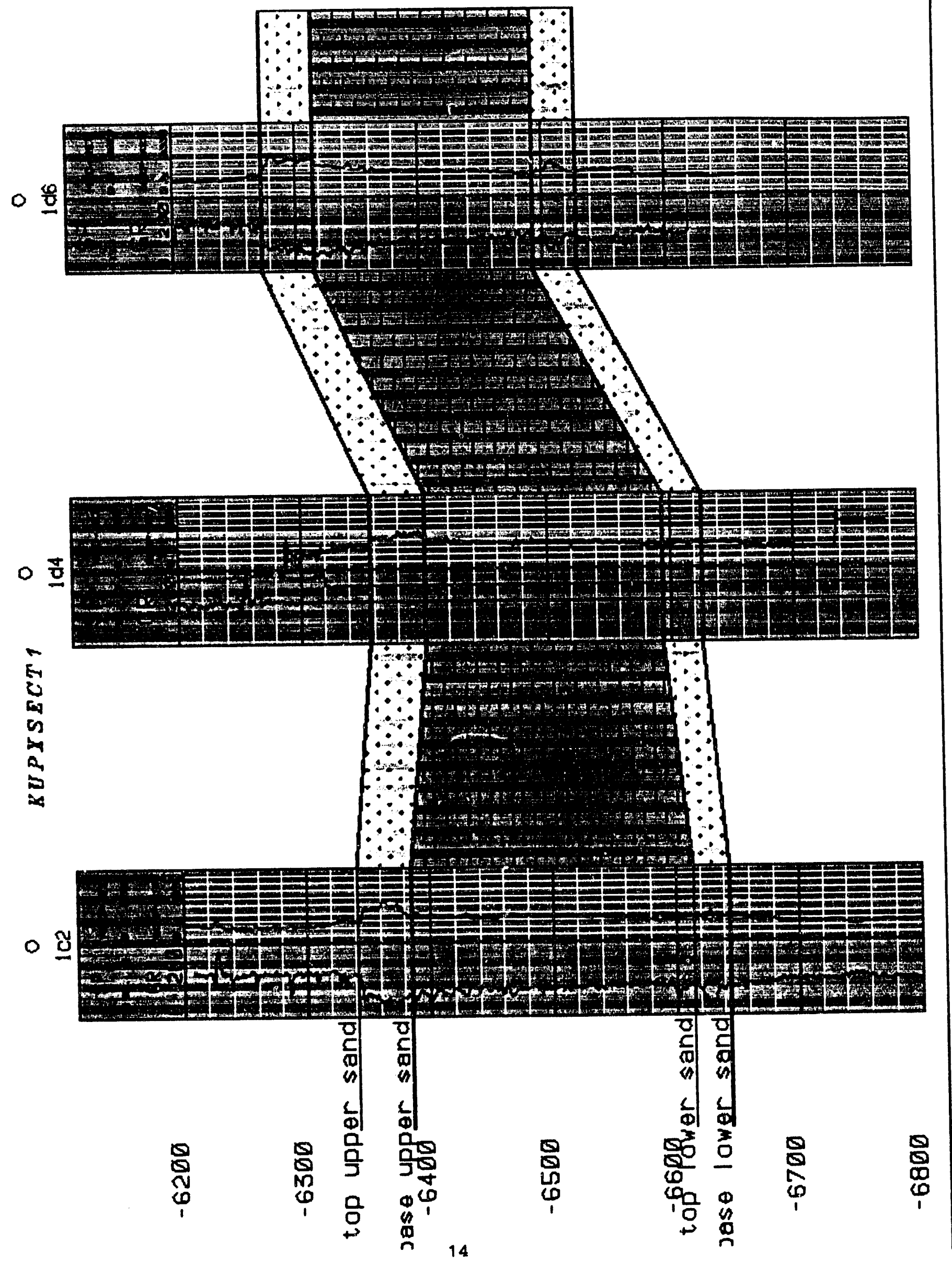


Figure 8. Westerly Cotponent of Dip and

Inter-Sand Thinning Across Kuparuk Field.

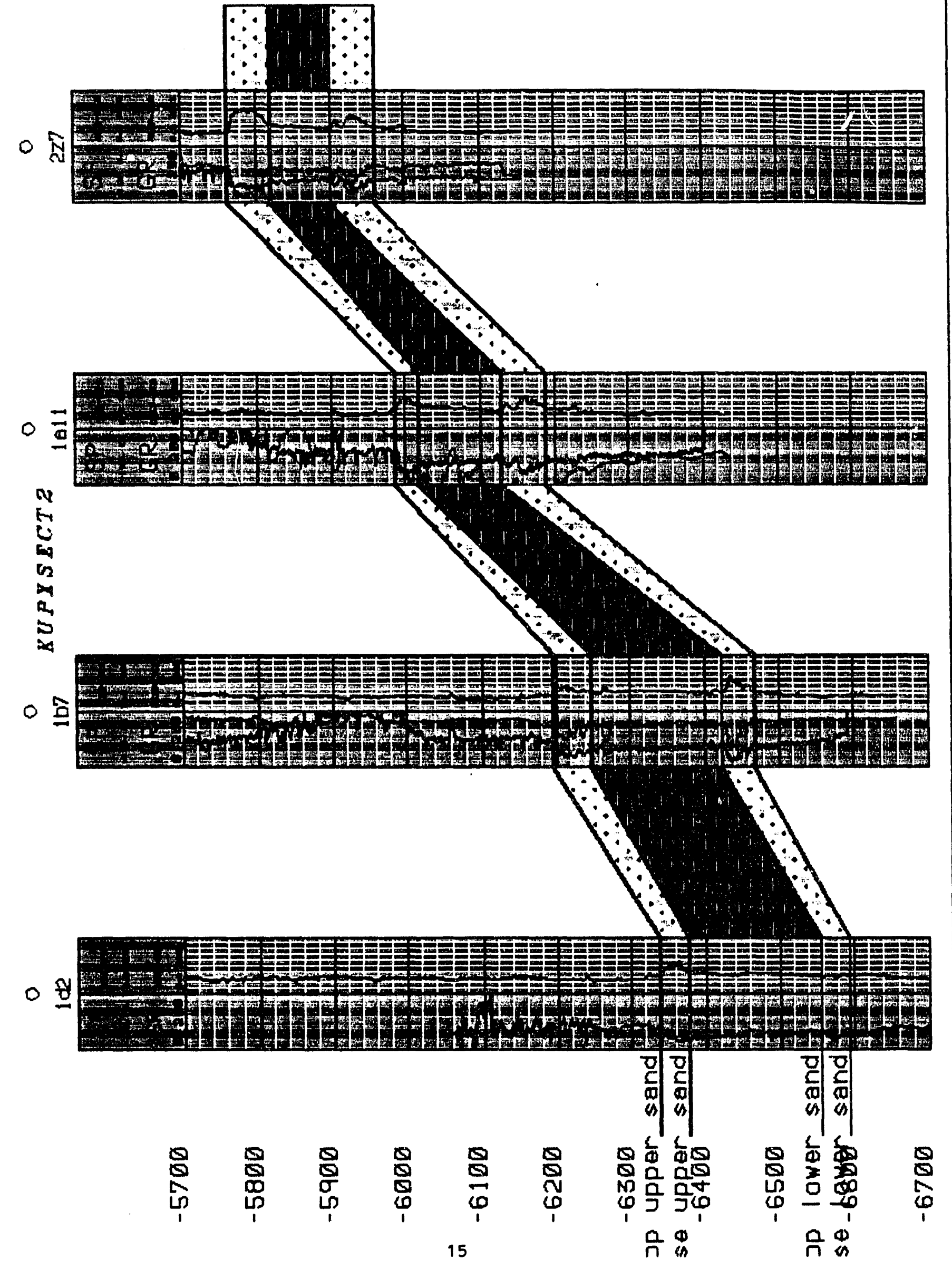


Figure 9. Structural Cross Section Across Kuparuk Field (Masterson and Paris).

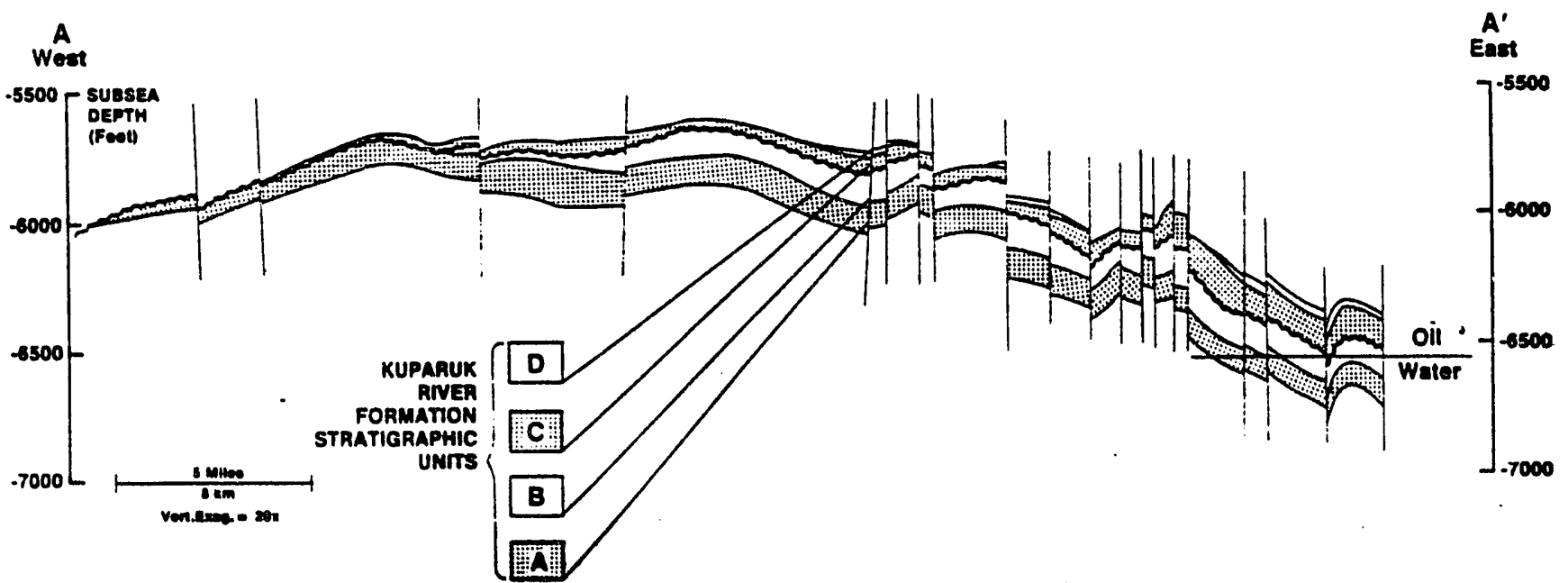

Figure 10. Faulting Within Kuparuk Field (Masterson and Paris).

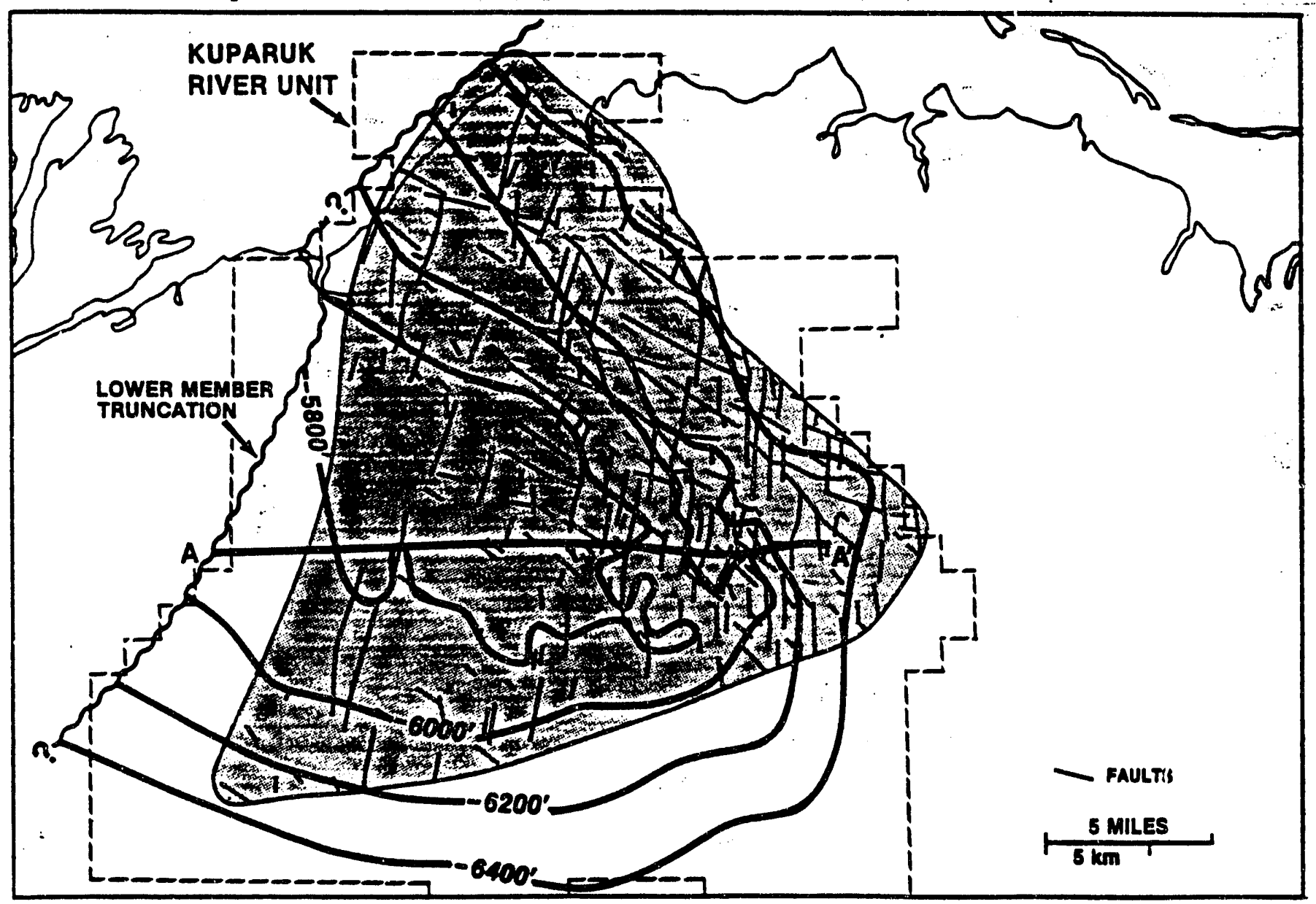




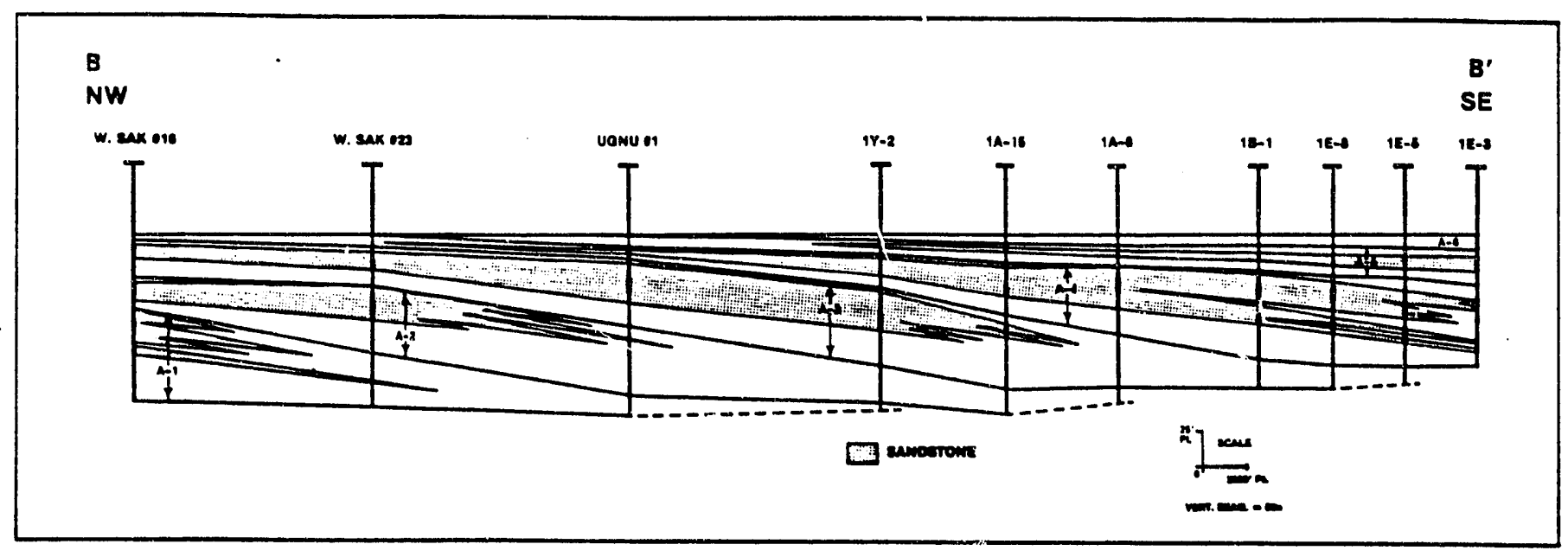

Figure 11. Stratigraphic Cross Section Across Kuparuk Field (Masterson and Paris). 


\section{RESERVOIR HETEROGENEITY CLASSIFICATION SYSTEM}

\section{FOR TORIS AND TORIS DATA BASE}

The work performed during this quarter as part of the Reservoir Heterogeneity Classification System for TORIS and TORIS data base included the compilation of production and injection data from the Alaskan oil fields and the compilation of geological and reservoir data. Following an extensive literature review, these data were collected, completed and plotted. Data not available in the literature have been requested from the field operators. Figures 12-15 display the monthly production and injection data for the Ivishak and Kekiktuk formations of Endicott oil field. Tables 3 and 4 include the reservoir heterogeneity and TORIS data base data for the Kekiktuk formation of Endicott oil field.

The collection of data for TORIS data base for all the Alaskan oil fields continues during the next quarter and will be included in the final report.

\section{RESERVOIR FLUID CHARACTERIZATION}

Under the reservoir fluid characterization task, work on two subtasks, namely: 1) modeling of asphaltene equilibria and prediction of asphaltene precipitation from Alaskan crude oils under influence of a miscible solvent; and 2) study of effect of asphaltene deposition on rock-fluid properties has been completed. Preliminary results on these tasks were provided in the last Technical Progress Report and all the final results will be provided in the Final Technical Report. 
Figure 12.

ENDICOTT ENDICOTT MONTHLY PRODUCTION DATA

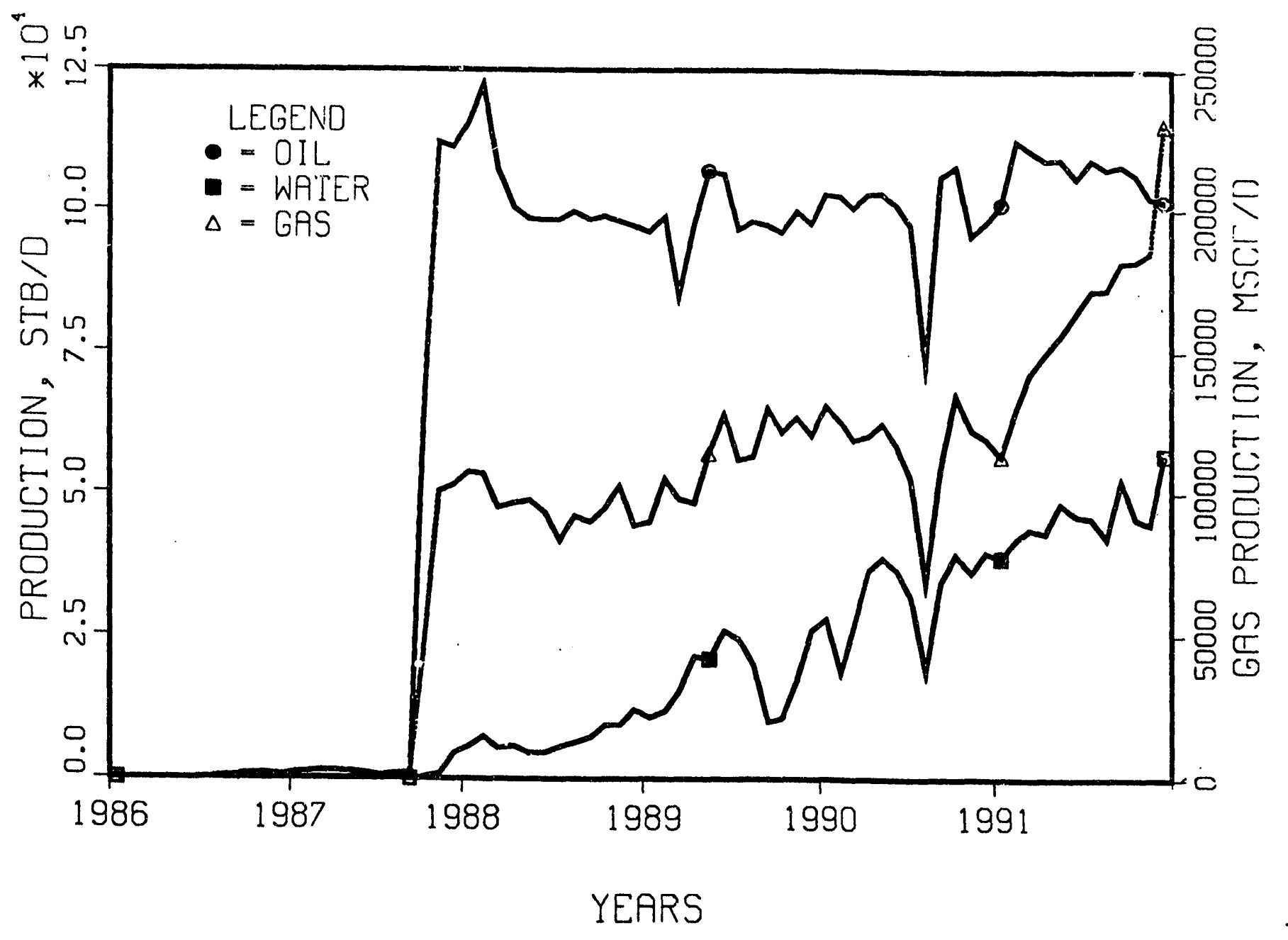


Figure 13.

ENDICOTT IVISHAK MONTHLY PRODUCTION DATA

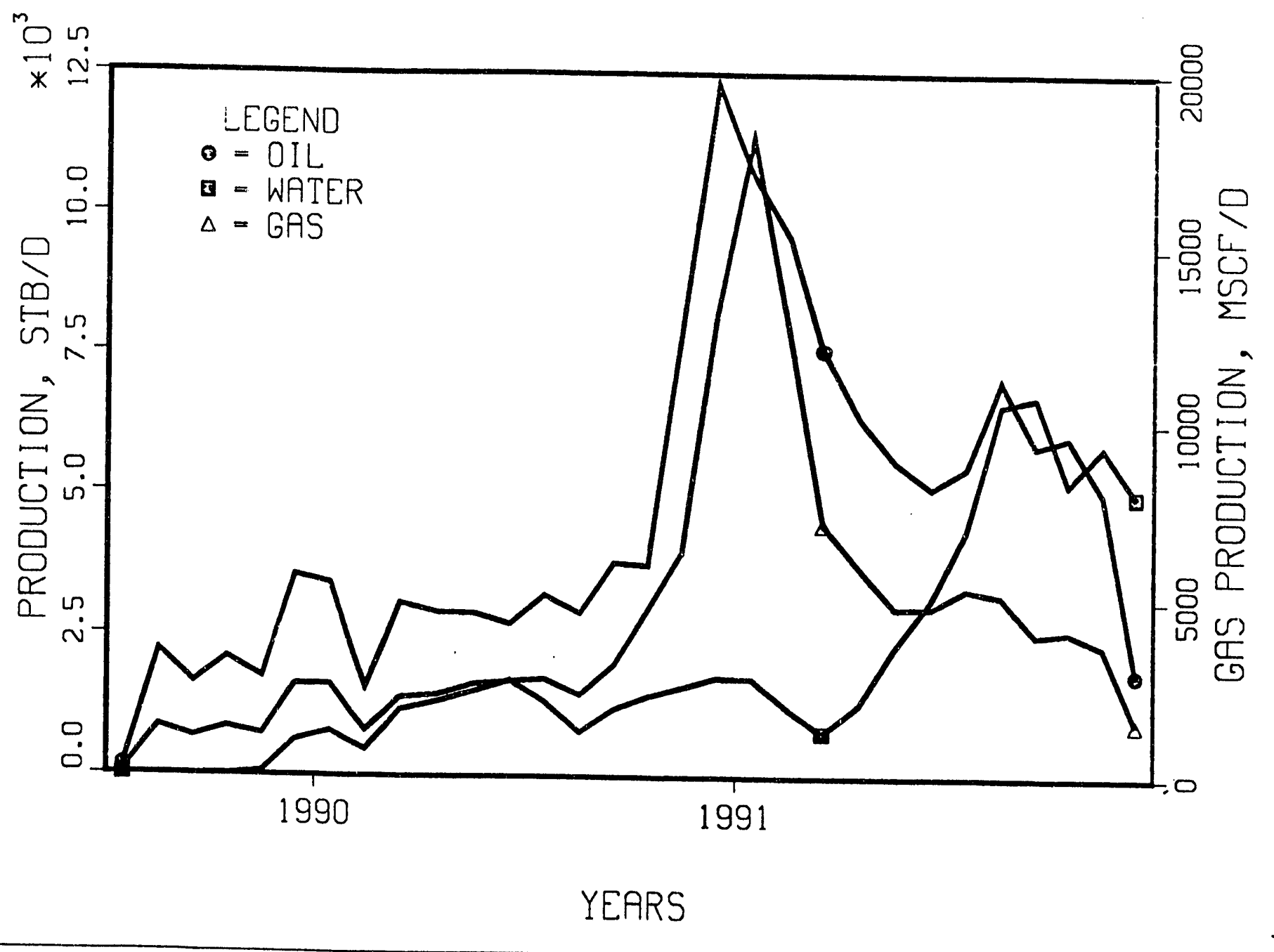


Figure 14.

ENDICOTT-ENDICOTI MONTHLY INJECTION DATA

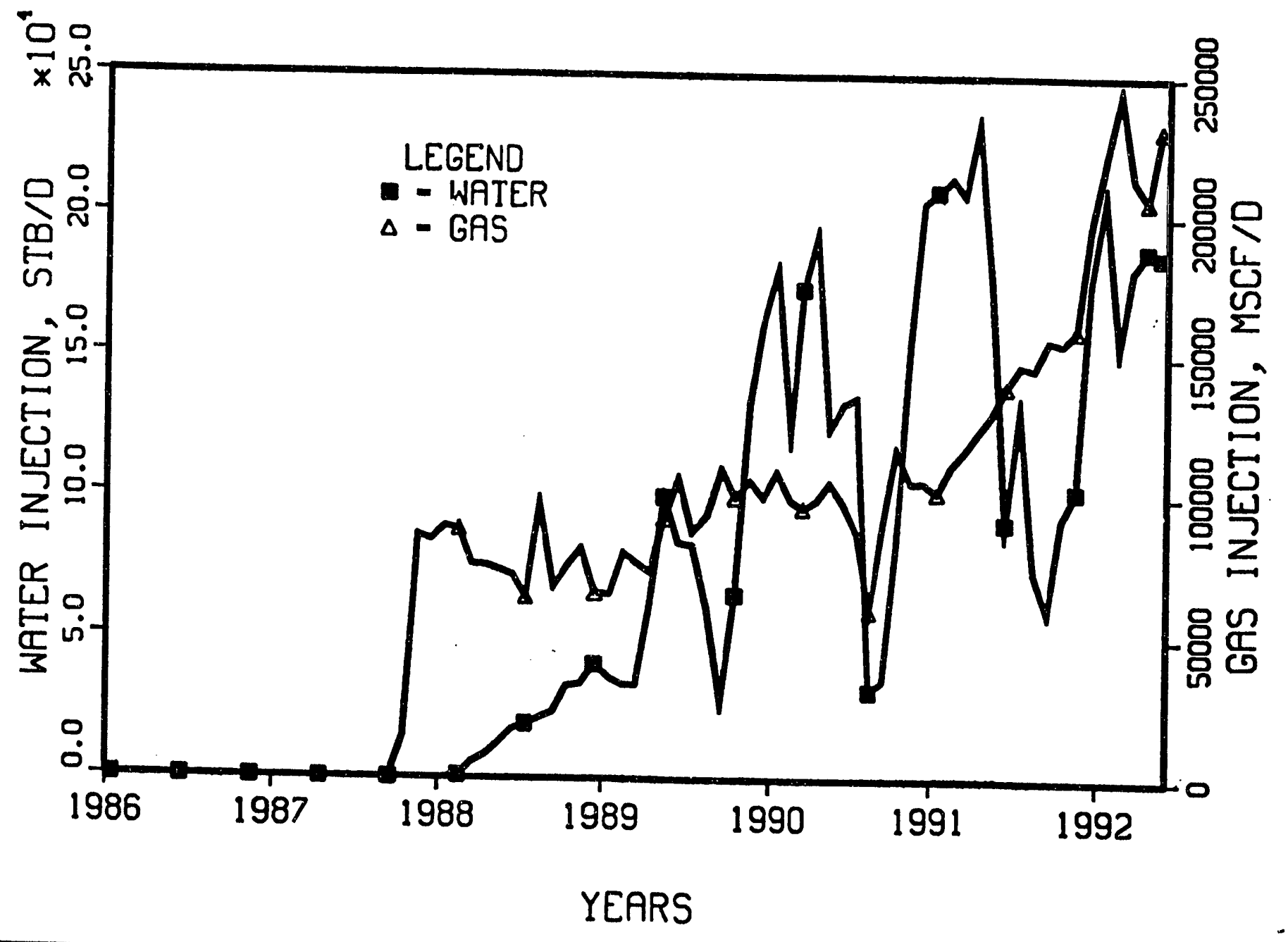


Figure 15.

ENDICOTT IVISHAK MONTHLY INJECTION DATA

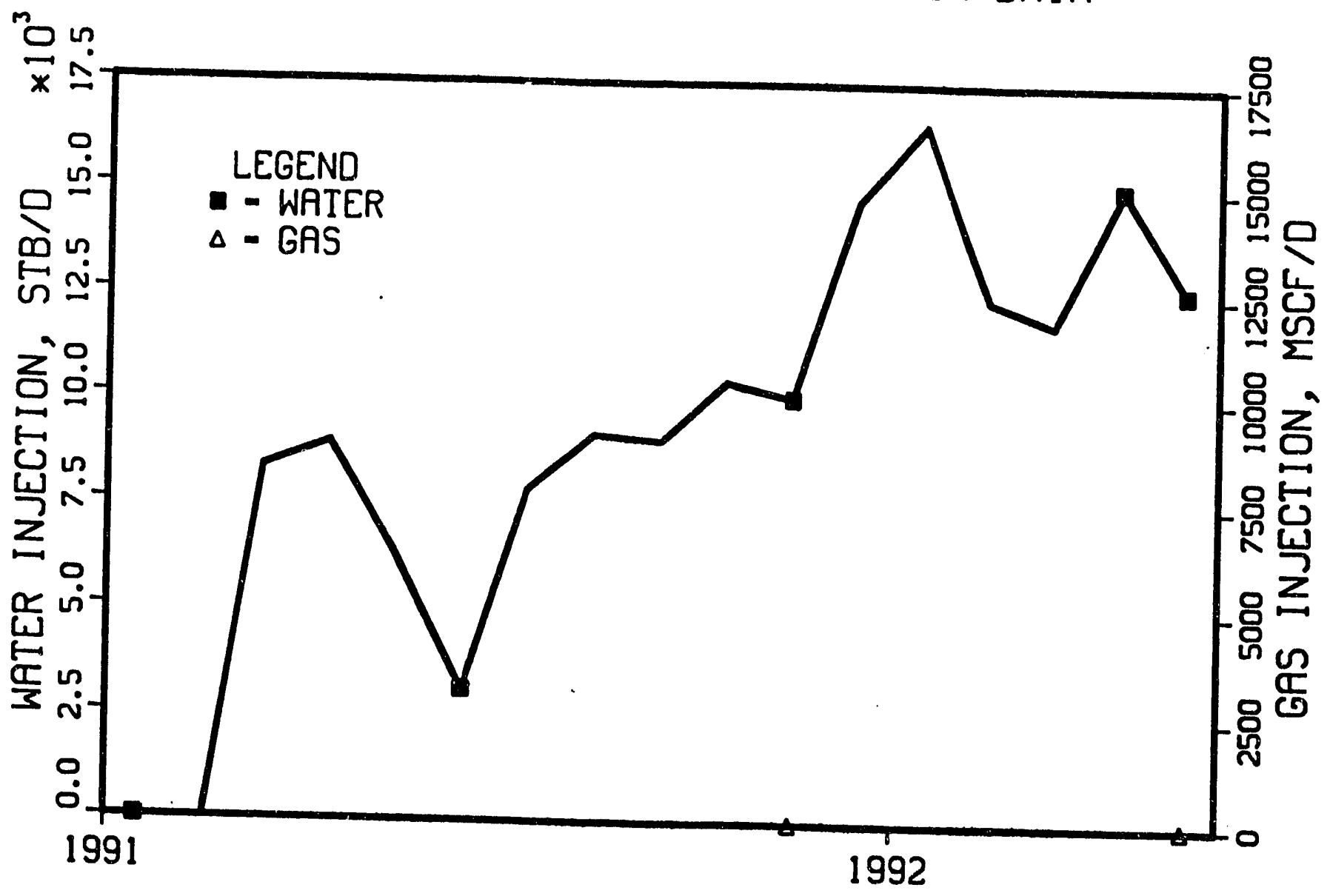

YEARS 


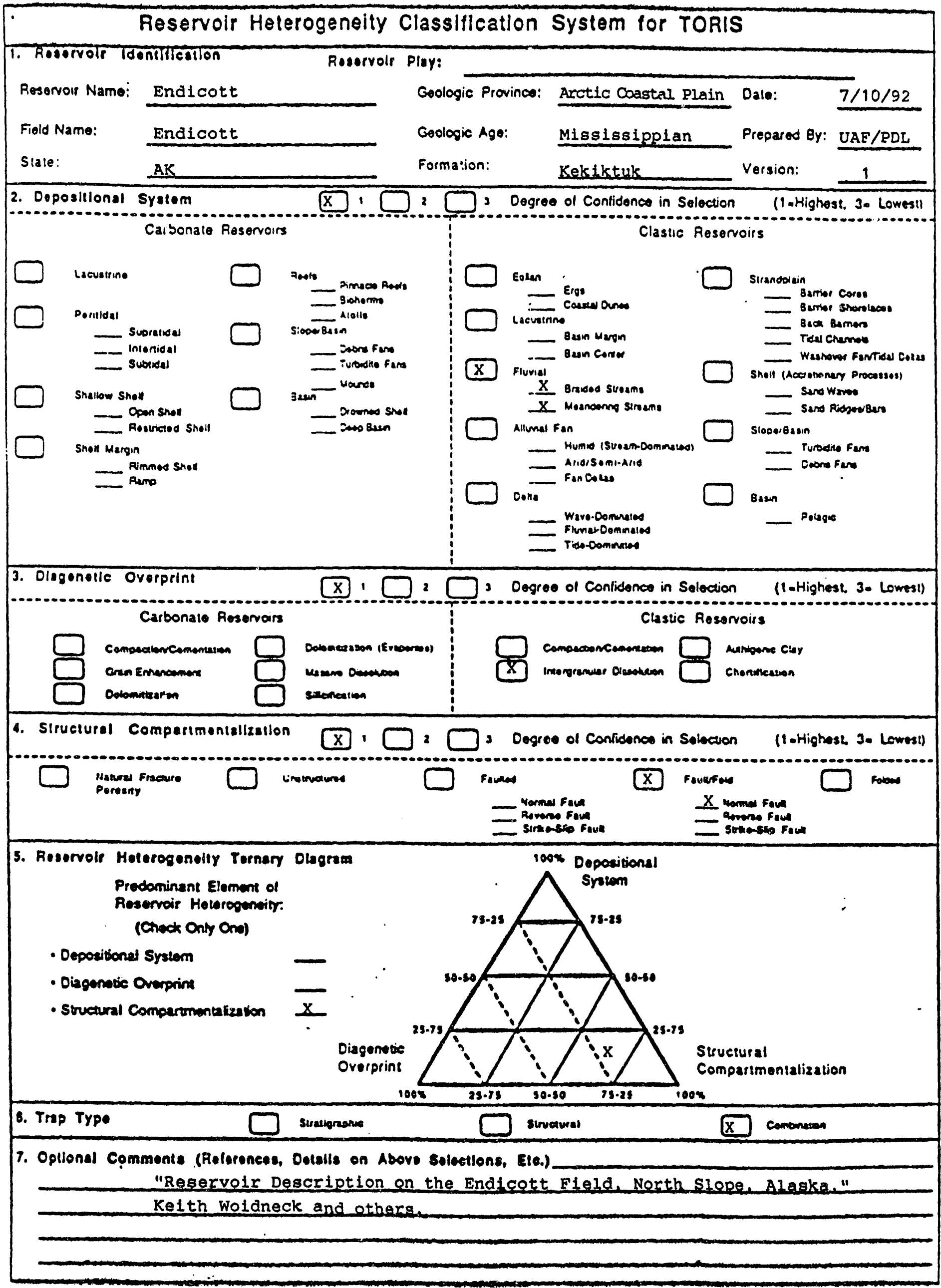




\section{TORIS DATA BASE \\ FIELD: ENDICOTT (KEKIKTUK)}

Record 1:

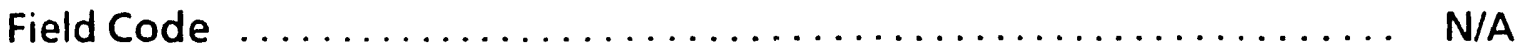

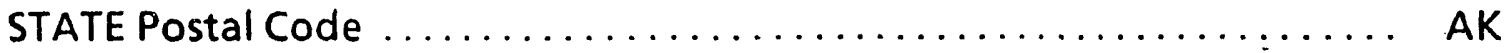

Lithology Code $(-1,0=$ Unknown; $1=S S, 2=L S ; 3=$ Dolo.) $\ldots \ldots \ldots \ldots \ldots 1$

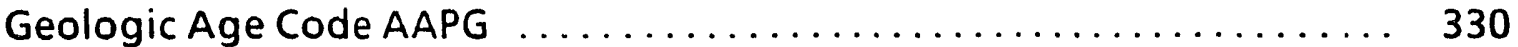

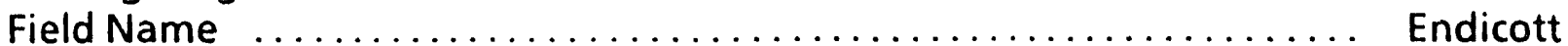

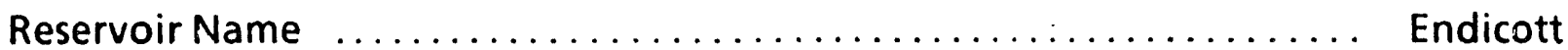

Reference Number $\ldots \ldots \ldots \ldots \ldots \ldots \ldots \ldots \ldots \ldots \ldots \ldots \ldots \ldots \ldots \ldots$

Load Number ................................... N/A

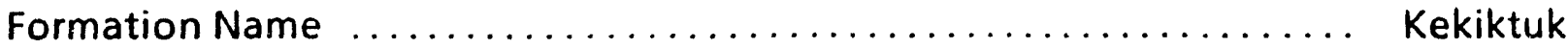

Record 2:

(1) Field Acres (Acres) $\ldots \ldots \ldots \ldots \ldots \ldots \ldots \ldots \ldots \ldots \ldots \ldots \ldots \ldots$

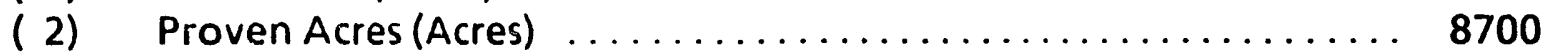

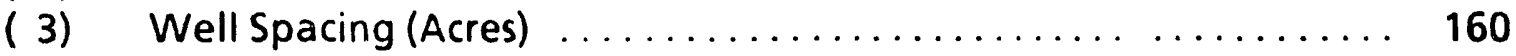

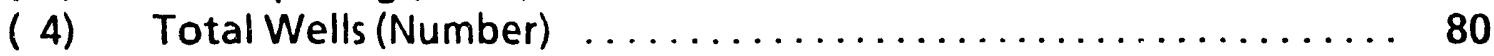

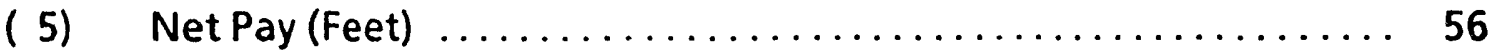

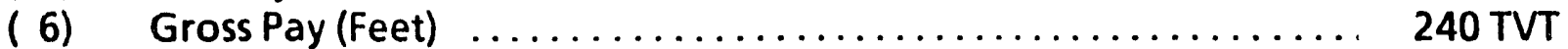

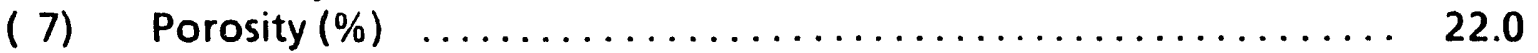

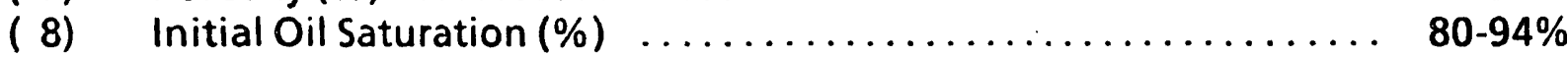

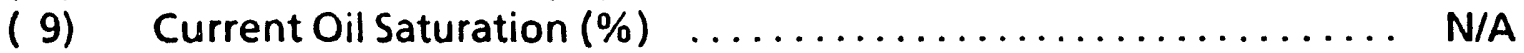

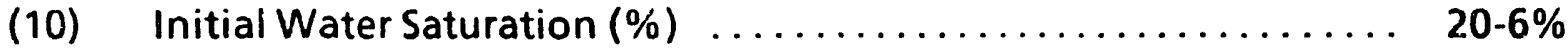

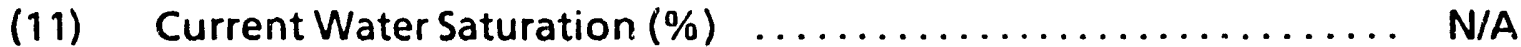

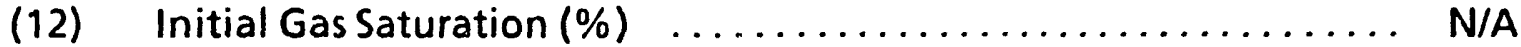

(13) Current Gas Saturation (\%) ....................... N/A

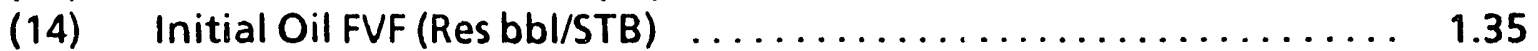

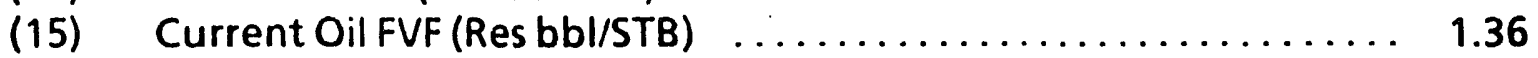

(16) True Vertical Depth (Feet)-.--Mid-Perforation ............. 10000.0

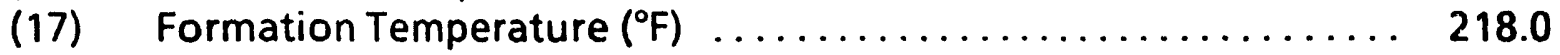

Record 3:

(18) Current Formation Pressure (PSI) $\ldots \ldots \ldots \ldots \ldots \ldots \ldots \ldots \ldots \ldots \ldots$

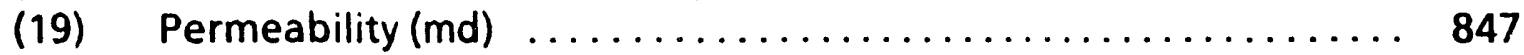

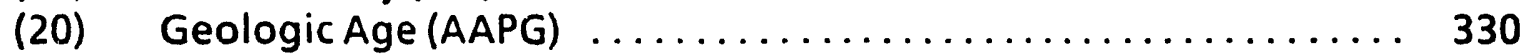

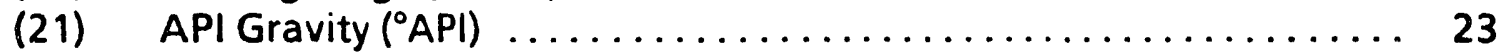

(22) Oil Viscosity (CP) :e Reservoir condition $\ldots \ldots \ldots \ldots \ldots \ldots \ldots \ldots \ldots$

(23) Formation Salinity (ppm TDS) ....................... 28000

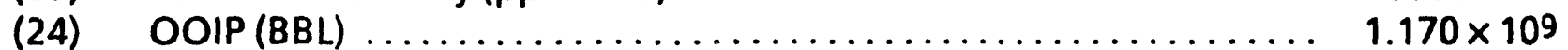

(25) Primary Recovery Factor (Fraction of OOIP) $\ldots \ldots \ldots \ldots \ldots \ldots \ldots$

(26) Cumulative Oil Production (BBL) $\ldots \ldots \ldots \ldots \ldots \ldots \ldots \ldots \ldots \ldots \ldots \ldots \ldots \ldots$ 
(27) Year For Cumulative Oil Production; EX/1986 ............. 1990

(28) Technicai Availability Date (Year); Ex/1990 ............... N/A

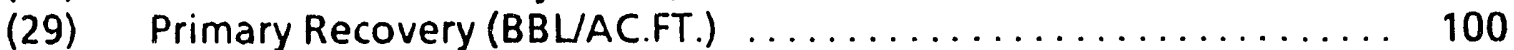

(30) Primary Recovery (BBL)

$115.5 \times 106$

Year For Primary Recovery

1982

Current Producing GOR (SCF/BBL)

1220.0

Initial Producing GOR (SCF/BBL)

763.0

Record 4:

(34) Reservoir Acreage (Acres)

(35) Initial Formation Pressure (PSI)

8700

Reservoir Dip (DEG)

4890 psi @ 10,000 TVDSS

Production Wells (Number)

5.5

Injection Wells (Number)

61

(39)

(40)

Swept Zone Oil Saturation (\%) (Residual to Water)

18

(41)

Injection Water Salinity (ppm TDS)

N/A

Clay Content (\%)

(42)

Dykstra-Parsolis Coefficient

N/A

(43)

Current Injection Rate (B/DNWELL)

Trace $-5 \%$

N/A

(44)

Fractured-Fault $(Y, N)(N=0, Y=1)$

10,000

(45)

(46)

(47)

(48)

(49)

Shale Break or Laminations $(Y, N)(N=0, Y=1)$

Major Gas $\operatorname{Cap}(Y, N)(N=0, Y=1)$

Field Multiplier (Number)

RRC Dist ict

N/A

N/A

Production Rate (MBBL/D); For the Year shown in element $28 \ldots$.

100.0

Recovery Efficiency-Waterflood (Factor)

$67 \%$

(51) Ultimate Recovery Factor (Fraction of OOIP)

(Primary Plus Secondary)

$45 \%$

Record 5:

(52) Geologic Play Code (Table II)

(53) Depositional System Code (Table III) .................... 130

(54) Depositional System Degree of Confidence

( 1 = Highest, $2=$ Moderate, 3 = Lowest) $\ldots \ldots \ldots \ldots \ldots \ldots \ldots \ldots$

(55) Diagenetic Overprint Code (Table IV) ................ 7

(56) Diagenetic Overprint Degree of Confidence ( 1 = Highest, 2 = Moderate, 3 = Lowest) $\ldots \ldots \ldots \ldots \ldots \ldots \ldots \ldots$

Structural Compartmentalization (Table V)

Structural Compartmentalization Degree of Confidence ( 1 = Highest, 2 = Moderate, 3 = Lowest)

Predominant Element of Reservoir Heterogeneity

( 1 = Depositional System, 2 = Diagenetic Overprint, 3 = Structural Compartmentalization) $\ldots \ldots \ldots \ldots \ldots \ldots \ldots \ldots$

Trap Type (1 = Stratigraphic, 2 = Structural, 3 = Combination) ... 3

Geologic Province (Table VI) ................... 058 

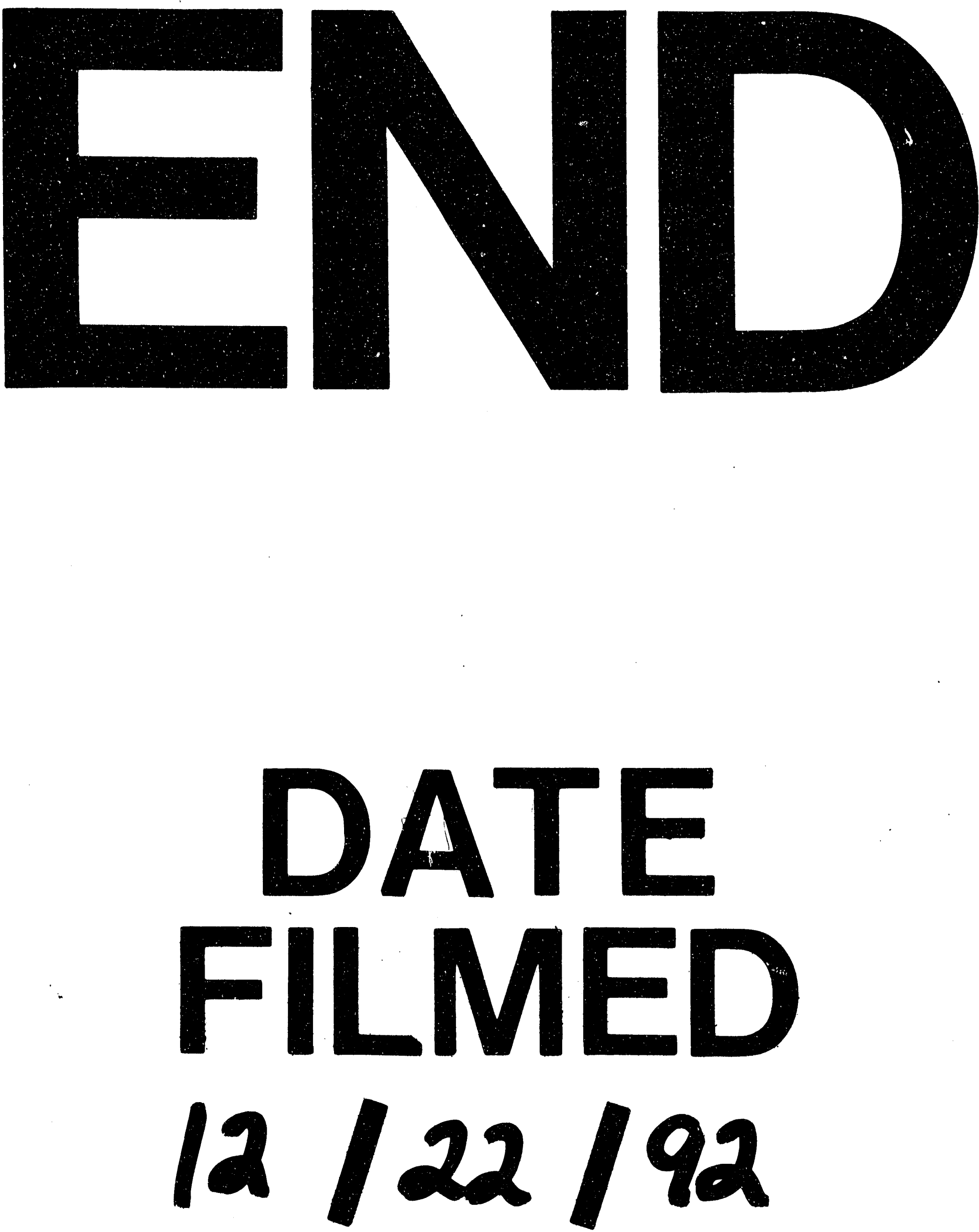